\title{
Systemic therapies for severe atopic dermatitis in children and adults
}

ARTICLE in THE JOURNAL OF ALLERGY AND CLINICAL IMMUNOLOGY · SEPTEMBER 2013

Impact Factor: 11.48 · DOI: 10.1016/j.jaci.2013.03.016 · Source: PubMed

2 AUTHORS, INCLUDING:

\section{Alan Irvine}

Trinity College Dublin

204 PUBLICATIONS 8,465 CITATIONS

SEE PROFILE 


\section{Maintenance of Certification Clinical Management Series}

3

4 Title: Systemic therapies for severe atopic dermatitis in children and adults

5

6 Carsten Flohr MD $\mathrm{PhD}^{1}$ and Alan D. Irvine MD FRCPI $2,3,4$

7 1Department of Paediatric Dermatology, St John's Institute of Dermatology, Guy's and St

8 Thomas' Hospitals NHS Foundation Trust and King's College, London, UK

9 2National Children's Research Centre Our Lady's Children's Hospital, Crumlin, Dublin

$10{ }^{3}$ Department of Pediatric Dermatology Our Lady's Children's Hospital, Crumlin, Dublin

$11 \quad{ }^{4}$ Department of Clinical Medicine, Trinity College Dublin

12

13 Correspondence to: Carsten Flohr (carsten.flohr@kcl.ac.uk) or Alan D. Irvine

14 (irvinea@tcd.ie)

15 Phone : Carsten Flohr +447806514078; Alan Irvine +3531428 2532

16 


\section{Abbreviations}

18 ALL: Acute lymphoblastic leukemia

19 CDLQI: Children's Dermatology Life Quality Index

20 DLQI: Dermatology Life Quality Index

21 SCORAD: SCORing Atopic Dermatitis

22 TPMT: Thiopurine methyltransferase

23 6-MP: 6-Mercaptopurine

24 6-TG: 6-Thioguanine

25

26 Keywords

27 Atopic dermatitis

28 Eczema

29 Methotrexate

30 Cyclosporin

31 Azathioprine

32 Mycophenylate Moefetil

33

34

35 


\section{$37 \quad$ Clinical Vignette}

230 words

A 7 year old boy was referred to a tertiary paediatric dermatology center with a lifelong history of severe atopic dermatitis (AD). He had lately missed several weeks from school due to hospital admissions for recurrent skin infections, lack of sleep and intense discomfort. Over the years, he had primarily been on regular emollients, potent topical steroids and more recently failed a trial of UV phototherapy. Intense topical steroid therapies, wet wraps with emollient over night, after treatment education on the day ward, and a prolonged course on oral antibiotics did not result in sustained improvement. The history did not suggest any contributory immediate or delayed allergies, and skin prick testing to a broad range of food and aeroallergens as well as patch testing to a range of relevant allergens were negative. Due to the severity of his disease (SCORAD 60/103; CDLQI 28/30), he was started on cyclosporine. A dose of up to $5 \mathrm{mg} / \mathrm{kg} /$ day somewhat decreased disease severity over a 12 week period but his quality of life was still significantly affected (SCORAD decreased to 32; CDLQI: 18). Our patient was subsequently switched to azathioprine at $3 \mathrm{mg} / \mathrm{kg} /$ day in 2 divided doses, with no significant clinical benefit after 3 months. Finally, he was commenced on methotrexate at a treatment dose of $0.4 \mathrm{mg} / \mathrm{kg} /$ week. Folic acid was also given (5 mg OD except the day of his MTX dose); 12 weeks later, his skin inflammation had settled (SCORAD: 10; CDLQI: 4), and he had missed no days off school in 4 weeks. At review after 6 months, he remained well and was using minimal topical therapies. His SCORAD continued to be low at 8 and his CDLQI was 3. 
Review

63

64

65

66

67

68

69

70

71

72

73

81

Severe AD has a very significant impact on the quality of life of the affected patient and, in children, additionally on the family unit; posing a considerable therapeutic challenge to the physician, as illustrated in this case report. In general, mild-to-moderate AD can be adequately controlled with topical and/or UV therapy. However, a subset of children and adults with severe or recalcitrant disease require systemic immuno-suppression to induce and maintain disease control. ${ }^{1}$

\section{What is severe AD?}

There is no universally agreed definition of severe AD. From a clinical point of view, severe disease can be thought of as AD that is resistant to potent topical corticosteroid or calcineurin inhibitor and UV therapy and that is associated with a considerable impact on quality of life. The European Academy of Dermatology \& Venereology Taskforce on AD defined severe disease as having a SCORAD severity score $>40$, but this definition is not very helpful when faced with a case in clinic, where the decision to start immuno-suppressive treatment is not only guided by disease severity but also the impairment of the patient's quality of life, for instance with regard to sleep disturbance and impact on schooling. ${ }^{2}$

\section{Using systemic immuno-suppressive drugs in children and adults with severe AD}


83 Before considering commencing a child or adult on an immuno-suppressive drug, it is important to identify potential triggers, such as irritants, and exacerbating factors (such as immediate and delayed hypersensitivity) through allergy testing (skin prick testing, specific IgE as well as patch testing). ${ }^{3}$ In addition, reasons why topical treatments have failed need to be taken into account. Applying creams and ointments is labour intensive and time consuming. Patient education on their use has a proven additional benefit with regard to both disease severity and quality of life. ${ }^{4}$ Sometimes, admitting a patient for a few days for education and intensive topical therapy can be helpful, and also can explore adherence to an agreed treatment plan. Furthermore, skin infection, in particular Staphylococcus aureus and less commonly Herpes simplex, can be main drivers of disease flares. It is therefore important to be vigilant during physical examination and to identify and treat skin infections where present. It is accepted practice to also use antimicrobial soap replacement in such situations, but there is only limited evidence that regular antimicrobial therapy (e.g. antimicrobial bath additives) as a prophylactic measure reduces the risk of disease flares. ${ }^{5}$ In young children, occlusive garments (wet wraps) are commonly used as a short-term therapy to reduce more generalised eczematous inflammation and some centres also use them for longterm treatment of severe cases. However, there is limited RCT evidence, long-term studies that evaluate systemic absorption and skin atrophy are lacking and their use is rather impracticable in adults. ${ }^{6}$

With regard to immuno-suppressive treatments, there is a paucity of published evidence to guide clinical practice, especially in children, and prescribing therefore has to be guided by experience in adults and the use of these drugs in other severe childhood inflammatory disorders, such as rheumatoid arthritis or inflammatory bowel 
disease. ${ }^{7}$ Not surprisingly therefore there is wide variation in treatment approaches amongst clinicians, as suggested by a recent survey among pediatric dermatologists in 8 European countries. ${ }^{8}$ In this survey, cyclosporine was the most utilised agent overall for treatment periods up to a year, while azathioprine and especially methotrexate are instituted less frequently, but for longer treatment courses. Oral gluco-corticosteroids are not recommended for long-term treatment because of the risk of diabetes, hypertension, gastric ulcers, osteoporosis, skin atrophy and, in children, detrimental effects on growth. ${ }^{9}$ A complicating factor is that systemic immuno-suppressive drugs are not licensed for use in severe AD, except Germany where cyclosporine A is approved for the management of severe AD in patients over 16 years of age. There is no officially agreed minimum age for the use of immuno-suppressive therapy in children and such considerations are to a degree arbitrary. Many physicians only use such agents in teenagers rather than younger children, but this is not based on robust evidence, and research with regard to drug safety in younger children and optimal dosing to maximize efficacy and minimise toxicity is clearly needed. ${ }^{7}$ Furthermore, which systemic is preferred as a first line varies, and depends not only on licensing considerations, but also on the individual clinical situation. For instance, where disease control needs to be achieved quickly, Cyclosporine would be the drug of choice. However, it is considered less suitable for long-term therapy due to its side effect profile. In such situations, treatment with either Azathioprine or Methotrexate could be preferable.

\section{Cyclosporine A}

Cyclosporine is a potent inhibitor of T-lymphocyte-dependent immune responses. A systematic review of 11 clinical trials suggested that it is an efficacious treatment but 
that relapse is rapid, once therapy is discontinued. ${ }^{10}$ The effectiveness of cyclosporine A was similar in children and adults, with better tolerability seen in younger patients. If cyclosporine is effective, remission is commonly seen within a few weeks. However, potential nephrotoxicity and hypertension limit its long-term use, and regular blood pressure and renal function measurements are therefore important. (See text boxes 1 and 2 for monitoring considerations for the use of systemic immuno-suppressants.)

\section{Azathioprine}

Azathioprine inhibits purine synthesis and thus proliferation of leucocytes. The target cells and mechanism of action in AD are not fully elucidated. ${ }^{11}$ Azathioprine has a complex metabolism with several immunosuppressant metabolites. The balance between thiopurine metabolites is governed by thiopurine methyltransferase (TPMT) activity, and the pre-treatment determination of TPMT genotype or activity level allows informed drug dosing to minimise myelotoxicity. Other side effects include headache and gastrointestinal upset, hepatotoxicity and drug hypersensitivity. There is concern about the potential long-term risk of lymphoma based on observations in inflammatory bowel disease, but the risk increase seen may be related to inflammatory bowel disease itself rather than be drug-related. ${ }^{12}$ More recently the emergence of progressive multifocal leukoencephalopathy (PML) in patients treated with azathioprine, either in combination with other immunomodulators, or as a single agent, has given further pause regarding this agent. A large scale ecological study of reported cases of PML in patients on immune suppression suggests that azathioprine appears to confer a significantly higher risk of PML compared to Cyclosporine (lower risk) or Methotrexate (minimal risk). These risks may be most relevant in the context of autoimmune disease 
and, to the best of our knowledge have not been reported in atopic dermatitis. ${ }^{13}$

157

158

Azathioprine has a slow onset of action, with clinical improvement sometimes only seen 8 weeks into therapy. Two double-blind, placebo controlled trials in adults with severe AD reported significant improvement in disease severity and quality of life..$^{2,9}$ More recently, a RCT comparing azathioprine and methotrexate in adults with severe AD suggested comparable efficacy, but this trial $(n=42)$ was not adequately powered to demonstrate equivalence in efficacy between the two drugs. ${ }^{14}$

\section{Methotrexate}

As with azathioprine, the mechanism of action of methotrexate in $\mathrm{AD}$ is not fully understood, but it is known to have anti-inflammatory properties and to also reduce allergen-specific T cell activity. ${ }^{1}$ Gastrointestinal disturbance, in particular nausea, liver function abnormalities and bone-marrow suppression are potential side effects, but the medication is generally well tolerated and considered safe in the long-term, partly based on rheumatology experience in children and adults. Onset of action is equally slow as seen with azathioprine. Subcutaneous administration may improve bioavailability and tolerability in patients who have either failed to respond to treatment or who suffer significant gastrointestinal intolerance. In addition to the RCT that compared methotrexate with azathioprine in adults, there has been one recent RCT in children $(\mathrm{n}=40)$, comparing methotrexate with cyclosporine, also suggesting equal treatment responses..$^{15}$ 
As no systemic treatment is universally safe and effective, several other systemic treatments have been tried over the years for recalcitrant AD. These include interferon gamma, mycophenylate mofetil, intravenous immunoglobulin and omaluzimab (a monoclonal IgE antibody). ${ }^{15}$ The evidence base for these treatments rests on case series or small open trials and is not sufficiently robust to guide clinical practice. ${ }^{1}$ Chinese herbal therapies, which showed early promise more than a decade ago, have since failed to gain a licence in the EU, USA or Japan.

\section{The case revisited}

Our patient with severe AD was treated with the three most commonly used systemic immuno-suppressants, only to eventually respond to methotrexate. We emphasise that this is an illustrative case to exemplify one child's treatment course and should not be interpreted as a comparative study or validated evidence on which to base therapeutic decisions. Given the significant impact on patients' quality of life and associated comorbidities, the current paucity of clinical trial evidence and new drug developments, in particular in children, is frustrating but equally understandable. Conducting drug trials in children is generally difficult because of licensing and safety issues. In addition, severe $\mathrm{AD}$ is a complex multiphase disease involving skin barrier impairment and multiple immunological pathways in the skin and systemically. The disease typically follows a waning and waxing pattern and is often compounded by skin infection. Small patient numbers with severe disease attending single centers make the disease 
series that have reported on the use of biologics in AD have shown limited effect on disease activity. ${ }^{16}$

The current state of affairs should not stop us from conducting clinical trials of existing agents. A good example of what can be done in a pediatric disease is acute lymphoblastic leukemia (ALL). A series of 4 clinical trials conducted by the UK Medical Research Council (MRC) demonstrated an increase in 12 year survival from 64\% to 83\%, achieved through maximising the efficacy and safety of existing therapies rather than the development of new drugs. ${ }^{17}$ ALL had, however, the benefit of good phenotyping, well defined outcome measures, including biomarkers, and a high priority

212 for research funding. From that point of view, we are moving forward in the right direction in $\mathrm{AD}$ research. For instance, the discovery of filaggrin loss-of-function mutations has provided an important genetic marker that may well influence therapeutic responses to systemic treatments, and this needs to be assessed in clinical trials. It will also be important to study whether any systemic immuno-suppressive agents alter the cytokine signatures of known T- and B-cell subsets both locally in the skin and systemically. As our understanding of the complex immunology of AD improves, new systemic drug targets may become available. Last but not least, further lessons regarding long-term side effects can be learned from solid organ transplant recipients, rheumatology and gastroenterology as well as patient registries. 


\section{Practical Learning Objectives:}

225

226

227

228
1. Be able to list common reasons why standard topical treatment may fail in patients with severe AD.

2. Have an understanding of the current evidence for the use of systemic immunosuppressive drugs in severe AD.

3. Be familiar with the recommended investigations prior to starting and during treatment with cyclosporine, azathioprine and methotrexate.

4. Be able to list important short and potential long-term side effects of cyclosporine, azathioprine, and methotrexate. 


\section{Text Box 1}

\section{Suggestions for baseline pre-treatment screening and other considerations}

for systemic immunosuppressant treatment in AD:

1. Pre-treatment infections screening can include VZV immune status, viral hepatitis screen, Mantoux/ImmunoSpot tuberculosis screening, HIV status and possibly HHV8 status, all depending on the population being treated. This requires local interpretation.

2. Pregnancy prevention should be considered when appropriate. FDA pregnancy categories are as follows: cyclosporine $\mathrm{C}$, azathioprine $\mathrm{D}$, mycophenylate mofetil D and methotrexate $\mathrm{X}$. This is relevant for any potentially pregnant female and should be understood by the prescriber and the patient and their family if relevant.

3. Live vaccines (e.g. MMR, Yellow fever, Typhoid, Smallpox) are contraindicated while taking cyclosporine, methotrexate and azathioprine.

4. Killed vaccines (e.g. influenza, hepatitis A, polio, rabies) may be less likely to induce immunisation in immunosuppressed individuals.

5. Immunosuppressed patients may have more severe forms of infections such as influenza, and these are therefore advised in patients on these therapies. Annual influenza vaccine is recommended.

6. Pneumococcus vaccines are recommended approximately every 5 years, guided by relevant antibody titres.

7. Patients and parents should be educated on sun behaviours while on immunosuppressants due to an increased risk of skin cancer.

8. Vitamin D levels should be checked before and during immunosuppressant treatments and supplemented as necessary. (Careful sun avoidance is widely recommended in immunosuppressed patients. Vitamin D deficiency is common in Northern climates and will be exacerbated by active sun avoidance.)

9. Each treatment has individual screening protocols for renal, hepatic and bone marrow impairment, and prescribers need to be familiar with these (see Textbox 2). 


\section{Text Box 2}

\section{Drug monitoring considerations for Cyclosporine A, Azathioprine, and Methotrexate.}

(Current monitoring practice varies between countries and centres and in children is primarily based on experience from other diseases (such as inflammatory bowel disease and rheumatoid arthritis). There are no official prescribing guidelines for the use of systemic therapies in children or adults with $\mathrm{AD}$. It is good practice to monitor treatment response with validated severity scores.)

\section{Cyclosporine A}

Dose: initially $2.5 \mathrm{mg} / \mathrm{kg}$ daily, increase to $\mathrm{max} 5 \mathrm{mg} / \mathrm{kg}$ daily in exceptional circumstances Drug monitoring: FBC, renal and liver profile as well as blood pressure at baseline, then fortnightly for first 2 months, then at least every 3 months.

\section{Azathioprine}

Dose: initially $1 \mathrm{mg} / \mathrm{kg} /$ day, increase to $\max 3 \mathrm{mg} / \mathrm{kg} /$ day, taking account of TPMT result TPMT $<3 \mathrm{nmol} / \mathrm{h} / \mathrm{mL}$ - contraindicated, TPMT 3-8 $\mathrm{nmol} / \mathrm{h} / \mathrm{mL}$ - low dose $0.5-1 \mathrm{mg} / \mathrm{kg} / \mathrm{day}$, TPMT 8- $14.5 \mathrm{nmol} / \mathrm{h} / \mathrm{mL} 1-3 \mathrm{mg} / \mathrm{kg} /$ day, TPMT $>14.5 \mathrm{nmol} / \mathrm{h} / \mathrm{mL}$ consider $>3 \mathrm{mg} / \mathrm{kg} /$ day if no response Drug monitoring: FBC, renal, liver profile and TPMT levels at baseline, then weekly for first month, then once every three months. Increases in dose should be accompanied by weekly blood tests. Repeat blood count if severe throat infection of other signs of potential marrow suppression.

\section{Methotrexate}

Dose: initially $200 \mathrm{micrograms} / \mathrm{kg}$ once weekly increased to max 400 micrograms/kg once weekly, depending on response, test dose is usually given at start of therapy, followed by bloods a week later; subcutaneous route can be used if oral route is ineffective or nausea is severe; folic acid is given at least once weekly in conjunction with methotrexate ( $5 \mathrm{mg}$ weekly, on different day). However, folic acid regimens vary and evidence is not conclusive as to which is most efficacious. Drug monitoring: FBC, renal and liver profile, CXR, Procollagen III (only in adults, as unreliable in growing children) at baseline, then forthightly for first month and at this frequency after each dose change.

\section{References}

Amor KT, Ryan C, Menter A. The use of cyclosporine in dermatology: part I. J Am Acad Dermatol 2010;63:925-46.

Amor KT, Ryan C, Menter A. The use of cyclosporine in dermatology: part II. J Am Acad Dermatol 2010;63: 949-72.

Meggitt SJ, Anstey AV, Mohd Mustapa MF, Reynolds NJ, Wakelin S. British Association of Dermatologists' guidelines for safe and effective prescribing of azathioprine 2011. Br J Dermatol 2011;165:11-34.

Shen S, O'Brien T, Yap LM, Prince HM, McCormack CJ. The use of methotrexate in dermatology: a review. Australasian J Dermatol 2012;53:1-18. 
Legend to Figure

237

238 Figure $1 \mathrm{a}$ and $\mathrm{b}$. Severe AD can have a significant impact on the lives of sufferers.

239 Despite potent topical and antimicrobial treatment, this 12 year old had extensive AD.

240 He finally got better on Methotrexate, having previously also failed to respond to

241 Cyclosporine and Azathioprine. 
a. ... has a widely agreed case definition.

b. ... has a strong evidence base on which to base treatment.

c. ... in some cases responds to methotrexate therapy.

d. ... is not treated with ultraviolet light therapy.

\section{Annotation:}

249 There are no widely agreed definitions of what constitutes severe AD but a combination of a validated measure of severity, such as the SCORAD or the EASI, in combination with a validated measure of quality of life scale (eg DLQI or CDLQI in children) can help monitor response to treatment, even in the clinic setting. Physician Global Assessment and Patient Global Assessment may also be recorded on a linear scale. Clinical photography may be a useful adjunct to monitor response to therapy. As discussed in the article, the available evidence to guide these decisions is not extensive. Narrow band or PUVA light therapy is helpful in some cases of severe AD, but is certainly not

257 universally effective and concern about skin cancer is real, especially where patients have fair skin and may be later on started on a systemic immuno-suppressive agent.

\section{Recommended further reading:}

260 McAleer MA, Flohr C, Irvine AD. Management of difficult and severe eczema in 261 childhood. Brit Med J 2012:e4770. 
a. Check TPMT activity and genotype before starting methotrexate.

b. Patient education regarding active skin care and topical therapy is unlikely to be helpful.

c. Check renal function before starting methotrexate.

d. Check blood pressure before starting azathioprine.

\section{Annotation:}

TPMT is an important enzyme involved in metabolism of azathioprine and 6-MP. TPMT

activity is genetically determined and can be tested prior to commencing therapy with these drugs. It is not relevant for methotrexate metabolism. Before consideration of immuno-supression, care should be taken to maximise topical therapies, including intensive education, and/or admission to day or inpatient wards. $90 \%$ of methotrexate excretion is via the renal route, approximately $10 \%$ is by biliary elimination. Azathioprine has no effect on blood pressure.

\section{Recommended further reading:}

Denby KS, Beck LA. Update on systemic therapies for atopic dermatitis. Curr Opin Allergy Clin Immunol_2012;12:421-6. 
Question 3. Cylosporine ...
a. ... takes up to 12 weeks before reaching full therapeutic effect.
b. ... can be safely co-prescribed with clarithromycin.
c. ... in theory does not increase the risk of lymphoma.

$$
\text { d. ... bioavailability may decrease if red wine is consumed beforehand. }
$$

\section{Annotation:}

Cylosporine, when prescribed at appropriate doses for $\mathrm{AD}$, will generally have a therapeutic effect within a couple of weeks of administration. Co-administration of macrolides (such as troleandomycin, erythromycin, and clarithromycin) that are potent inhibitors of CYP450 3A4 can significantly increase the blood concentrations of cyclosporine, which is primarily metabolized by CYP450 3A4. There is also an increased risk of renal and neurotoxicity. However, azithromycin and dirithromycin are not believed to inhibit CYP450 3A4. There are insufficient data on cyclosporine in AD to accurately rate lymphoma risk but data exist in other patient populations (eg solid organ transplant recipients) to suggest that this is a potential concern with longer term therapy. Red wine taken prior to cyclosporine administration may decrease bioavailability, possibly due to an effect on gut wall CYP450 3A4, and avoidance is therefore recommended.

\section{Recommended further reading:}

Amor KT, Ryan C, Menter A. The use of cyclosporine in dermatology: part I. J Am Acad Dermatol 2010;63:925-46.

Ryan C, Amor KT, Menter A. The use of cyclosporine in dermatology: part II. J Am Acad Dermatol 2010;63:949-72. 

systemic immuno-suppressive treatments for AD:

a. Avoid all immunizations.

b. Avoid all alcohol.

c. Avoid excessive UV light exposure.

d. Avoid St. John's Wort as a complementary therapy.

Annotation:

While live vaccines should be avoided, killed vaccinations are not contraindicated while on these immuno-suppressants. Alcohol should be avoided with methotrexate therapy but can be consumed, in moderation, with the other immune suppressants, although there is a recommendation to avoid red wine with cyclosporine. All systemic immune suppressing drugs are associated with an increased risk of skin cancer. While this risk is almost certainly higher with azathioprine than other available options, excessive sun exposure should be avoided with all these immune suppressants. St. John's Wort has a drug interaction with cyclosporine but not azathioprine or methotrexate.

\section{Recommended further reading:}

331 Denby KS, Beck LA. Update on systemic therapies for atopic dermatitis. Curr Opin Allergy Clin Immunol 2012;12:421-6. childhood. Brit Med J 2012:e4770. 
a. ... may cause renal impairment.

b. ... may cause bone marrow failure.

c. ... does not cause photosensitivity.

d. ... is metabolised by methylenetetrahydrofolate reductase.

342

Annotation:

344 Cyclosporine can cause renal impairment but azathioprine does not. Bone marrow

345 failure is one potential major adverse effect of azathioprine and requires regular

346 monitoring. Pre-treatment TPMT measurement helps to calibrate this risk. Azathioprine

347 therapy causes incorporation of 6-thioguanine (6-TG) into DNA of dividing cells. 6-TG

348 DNA is damaged by UVA, causing photosensitivity. Blood 6-TG levels can be measured, 349 partly to assess treatment compliance. Methotrexate is metabolised by dihydrofolic acid 350 reductase.

Recommended further reading: Dermatologists' guidelines for the safe and effective prescribing of azathioprine 2011. 
1 McAleer MA, Flohr C, Irvine AD. Management of difficult and severe eczema in childhood. Brit Med J 2012:e4770.

${ }^{2}$ Darsow U, Wollenberg A, Simon D, Taieb A, Werfel T, Oranje A, et al. ETFAD/EADV eczema task force 2009 position paper on diagnosis and treatment of atopic dermatitis. Journal of the European Academy of Dermatology and Venereology : JEADV. $2010 ; 24(3): 317-28$.

1. ${ }^{3}$ Atopic Eczema in Children - Guideline Consultation: A Systematic Review of the Treatments for Atopic Eczema and Guideline for its Management. London: National Institute for Clinical Excellence (NICE). 2007. www.nice.org.uk/CG05.

${ }^{4}$ Staab D, Diepgen TL, Fartasch M, Kupfer J, Lob-Corzilius T, Ring J, et al. Age related, structured educational programmes for the management of atopic dermatitis in children and adolescents: multicentre, randomised controlled trial. Brit Med J 2006;332:933-8.

${ }^{5}$ Bath-Hextall FJ, Birnie AJ, Ravenscroft JC, Williams HC. Interventions to reduce Staphylococcus aureus in the management of atopic eczema: an updated Cochrane review. Brit J Dermatol 2010;163:12-26.

${ }^{6}$ Braham SJ, Pugashetti R, Koo J, Maibach HI. Occlusive therapy in atopic dermatitis: overview. J Dermatolog Treat 2010;21:62-72.

${ }^{7}$ Schmitt J, Schakel K, Schmitt N, Meurer M. Systemic treatment of severe atopic eczema: a systematic review. Acta Dermato-Venereologica 2007; 87:100-11.

8 Proudfoot F, Powell AM, Ayis S, et al. The European treatment of severe atopic eczema in children taskforce (TREAT) survey. Brit J Dermatol 2013; submitted. 
${ }^{9}$ Akdis CA, Akdis M, Bieber T, et al. Diagnosis and treatment of atopic dermatitis in children and adults: European Academy of Allergy and Clinical Immunology/American Academy of Allergy, Asthma and Immunology/PRACTALL Consensus Report. J Allergy Clin Immunol 2006;118:152-69.

${ }^{10}$ Schmitt J, Schmitt N, Meurer M. Cyclosporin in the treatment of patients with atopic eczema - a systematic review and meta-analysis. JEADV 2007;21:606-19.

11 Meggitt SJ, Gray JC, Reynolds NJ. Azathioprine dosed by thiopurine methyltransferase activity for moderate-to-severe atopic eczema: a double-blind, randomised controlled trial. Lancet 2006;367:839-46.

12 Masunaga Y, Ohno K, Ogawa R, et al. Meta-analysis of risk of malignancy with immunosuppressive drugs in inflammatory bowel disease. Ann Pharmacother 2007;41:21-8.

${ }^{13}$ Schmedt N, Andersohn F, Garbe E. Signals of progressive multifocal leukoencephalopathy for immunosuppressants: a disproportionality analysis of spontaneous reports within the US Adverse Event Reporting System (AERS). Pharmacoepi Drug Safety 2012;21:1216-20.

${ }^{14}$ Schram ME, Roekevisch E, Leeflang MM, Bos JD, Schmitt J, Spuls PI. A randomized trial of methotrexate versus azathioprine for severe atopic eczema. J Allergy Clin Immunol 2011;128:353-9.

15 El-Khalawany MA, Hassan H, Shaaban D, et al. Methotrexate versus cyclosporine in the treatment of severe atopic dermatitis in children: a multicenter experience in Egypt. Eur J Pediatr 2012 Nov 16. Early online publication.

16 Taieb A, Seneschal J, Mossalayi MD. Biologics in atopic dermatitis. JDDG 2012;10:1748. 
17 Mitchell C, Richards S, Harrison CJ and Eden T. Long-term follow-up of the United Kingdom Medical Research Council protocols for childhood acute lymphoblastic leukaemia, 1980-2001. Leukemia 2010; 24: 406-418. 


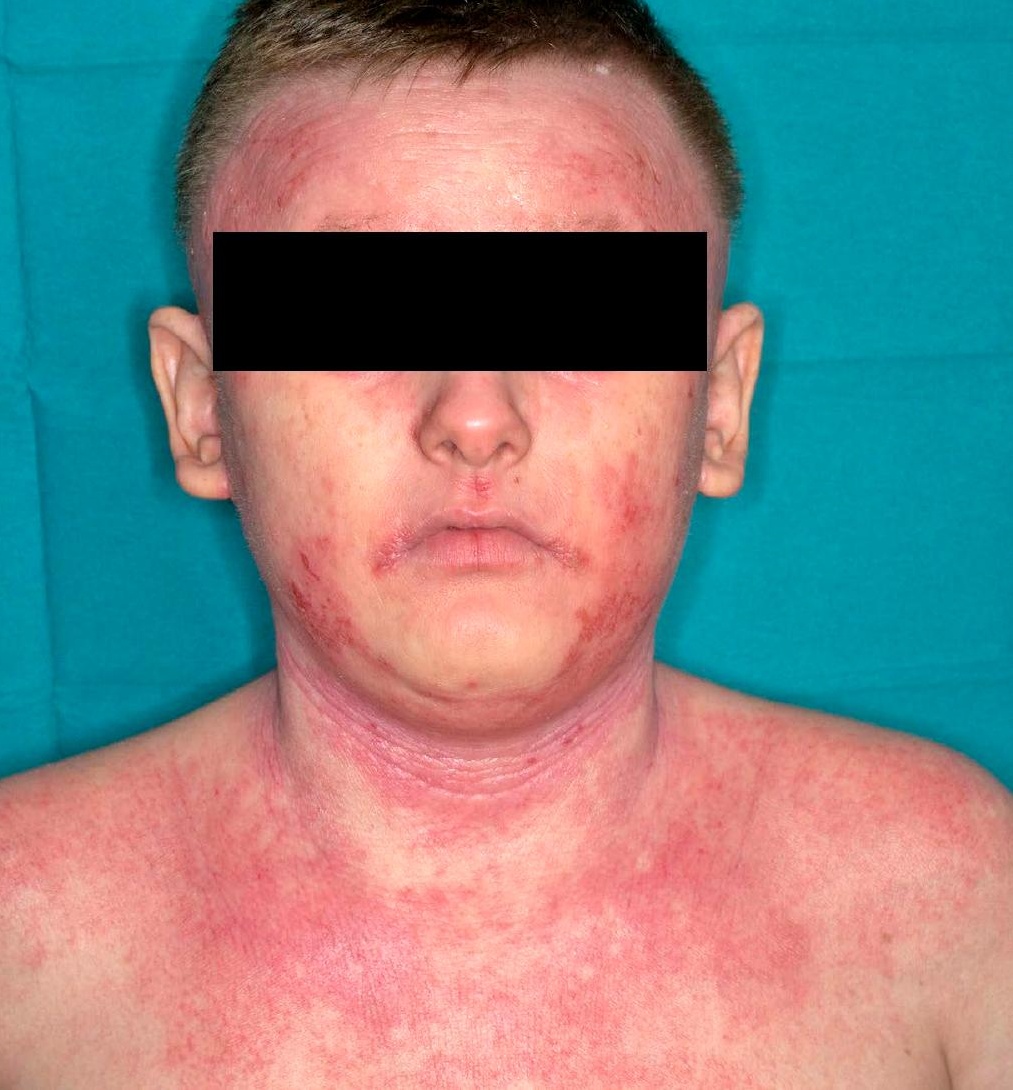

